\title{
HARMONIC ROUGH ISOMETRIES INTO HADAMARD SPACE*
}

\author{
PETER LI ${ }^{\dagger}$ AND JIAPING WANG ${ }^{\ddagger}$
}

0. Introduction. In a paper of Wan [W], he proved that for each holomorphic quadratic differential defined on the Euclidean 2-disk, $D^{2}$, there exists a harmonic diffeomorphism from the real hyperbolic plane $H_{\mathbb{R}}^{2}$ into itself, such that its Hopf differential is the given holomorphic quadratic differential after representing $H_{\mathbb{R}}^{2}$ by the Poincaré model. In addition, he also proved that this correspondence is one-to-one from the set of holomorphic quadratic differentials that are bounded with respect to the hyperbolic metric to the set of quasi-conformal harmonic diffeomorphisms from $H_{\mathbb{R}}^{2}$ onto itself. It is known in the literature that a quasi-conformal diffeomorphism of $H_{\mathbb{R}}^{2}$, when viewed as a quasi-conformal diffeomorphism of $D^{2}$, induces a quasisymmetric homeomorphism from $S^{1}$, the boundary of $D^{2}$, onto itself. Conversely, every quasi-symmetric homeomorphism of $S^{1}$ can be extended to a quasi-conformal diffeomorphism of $H_{\mathbb{R}}^{2}$ by identifying the geometric boundary of $H_{\mathbb{R}}^{2}$ with $S^{1}$. Inspired by all these, $\mathrm{R}$. Schoen $[\mathrm{S}]$ posed the following conjecture:

CONJECTURE 0.1. The set of quasi-symmetric homeomorphisms of $S^{1}$ has a oneto-one correspondence to the set of quasi-conformal harmonic diffeomorphisms of $H_{\mathbb{R}}^{2}$.

Specifically, this conjecture asserts that any quasi-symmetric homeomorphism of $S^{1}$ can be extended uniquely to a quasi-conformal harmonic diffeomorphism of $H_{\mathbb{R}}^{2}$. In [L-T 3], Tam and the first author confirmed the uniqueness part of this conjecture. They showed that if two quasi-conformal harmonic diffeomorphisms of $H_{\mathbb{R}}^{2}$ share the same boundary map, then they must be identical. Combining with Wan's theorem, the mapping which sends a bounded holomorphic quadratic differential to the quasisymmetric homeomorphism of $S^{1}$ - via the quasi-conformal harmonic diffeomorphism - is injective. Their argument relied on a precise version of the Bochner formula concerning the distance function between two harmonic maps and the fact that the energy density has a uniform positive lower bound for a quasi-conformal harmonic diffeomorphism of $H_{\mathbb{R}}^{2}$. However, it is unclear whether the last fact remains valid in higher dimension.

In a recent joint paper of Tam and Wan [T-W], they proved that this mapping is analytic and its image is open. On the other hand, surjectivity of this mapping still remains an open question. It is worth pointing out that a successful verification of the surjectivity will give a parametrization of the universal Teichmüller space by the space of bounded holomorphic quadratic differentials (see [T-W]).

In general, the Dirichlet problem at infinity for proper harmonic maps between two real hyperbolic spaces has been extensively studied by Tam and the first author in a series of papers [L-T 1], [L-T 2] and [L-T 3]. When the boundary map is $C^{1}$ and has nowhere vanishing energy density, they obtained rather complete and satisfactory results concerning the existence, uniqueness and regularity of the proper harmonic extensions. Later, Donnelly [D 1, D 2] extended some of their results to arbitrary rank-1 symmetric spaces.

\footnotetext{
* Received December 5, 1997; accepted for publication July 10, 1998.

† Department of Mathematics, University of California, Irvine, Irvine, CA 92697-3875, USA (pli@math.uci.edu). Research partially supported by NSF grant \#DMS-9626310.

$\ddagger$ Department of Mathematics, Cornell University, Ithaca, NY 14853-7901, USA (jwang@math. cornell.edu). Research partially supported by NSF grant \#DMS-9504441.
} 
The purpose of this paper is to formulate a generalization of Schoen's conjecture to higher dimensional real hyperbolic spaces and other rank-1 symmetric spaces. A major advantage in dimension 2 over higher dimensions is that if the boundary map of a harmonic map from $H_{\mathbb{R}}^{2}$ onto itself is a homeomorphism, then the harmonic map must be a diffeomorphism. The lack of this phenomenon and other 2-dimensional properties of harmonic maps makes the class of quasi-conformal harmonic diffeomorphisms too restrictive in higher dimensions. It turns out that the notion of harmonic rough isometry is more natural than the notion of quasi-conformal harmonic diffeomorphism in dimension greater than 2. We would also like to point out that in his proof of the celebrated rigidity theorem, Mostow [M] made extensive use of pseudo-isometries which are slightly more restrictive than rough isometries. Though the two definitions are quite similar, there are however some subtle differences which are important in the course of development.

In the first section, we will develop some basic properties of rough isometries between two Hadamard spaces. Here a Hadamard space is a nonpositively curved and geodesically complete metric space. For metric spaces, we adopt the definition of curvature bounds in terms of triangle comparisons with the model spaces. The class of Hadamard spaces is considerably more general than Cartan Hadamard manifolds and includes many infinite dimensional objects such as Hilbert spaces and non-locally compact buildings. Throughout this paper, unless otherwise indicated, either the domain or the target metric space is assumed to have strictly negative curvature. This extra assumption is necessary for showing that a rough isometry induces a boundary homeomorphism between the geometric boundaries of the domain and the target spaces. Moreover, two rough isometries induce the same boundary map if and only if they are bounded distance from each other. When $M=N$ and it is a finite dimensional rank-1 symmetric space, combining with an argument of Mostow [M], we conclude that the induced boundary map of a rough isometry must be quasi-conformal over the corresponding division algebra $K$ associated with $M$. We refer the reader to [M] for the precise definition. It should be pointed out that when $K=\mathbb{R}$ or the symmetric space $M$ is the real hyperbolic space $H_{\mathbb{R}}^{n}$, this definition coincides with the standard one and the induced boundary map is quasi-conformal between the standard sphere $S^{n-1}$ and itself. In the following, when a map between the geometric boundaries of a rank-1 symmetric space is referred to be quasi-conformal, it is understood to be so over the corresponding division algebra $K$ associated with the symmetric space. Conversely, an argument by Tukia [T] can be applied to show that every such quasi-conformal map of the geometric boundary between a rank-1 symmetric space and itself can be extended into a quasi-isometry (hence a rough isometry). Restricting ourselves to rank-1 symmetric spaces, we post the following generalization to the conjecture of Schoen.

CONJECTURE 0.2. Let $M$ be a rank-1 symmetric space. Then every quasi-conformal map from the boundary at infinity of $M$ to itself can be extended uniquely to a harmonic rough isometry from $M$ to itself.

In view of the preceding results, the conjecture is equivalent to saying that there exists a unique harmonic map of bounded distance from every rough isometry of $M$. Certainly, the same question can be asked for more general domain and target spaces other than rank-1 symmetric space. When both the domain and target are the same higher rank irreducible symmetric space of noncompact type, this question admits a complete and affirmative answer. In fact, a recent result of Kleiner and Leeb [K-L] says that every rough isometry between such a space is of bounded distance away from 
an isometry which is clearly a harmonic map. Moreover, Theorem 2.7 implies that it is unique. To verify our claim that Conjecture 0.2 is a generalization of Conjecture 0.1 , we show (Theorem 1.8) that a harmonic map between the real hyperbolic plane $H_{\mathbb{R}}^{2}$ and itself is a quasi-conformal diffeomorphism if and only if it is a rough isometry. It is pertinent to point out that the existence part of Conjecture 0.2 is valid when the rank-1 symmetric space is either a quaternionic hyperbolic space or the Cayley plane. This follows immediately by the following theorem of Pansu [P 1], due to the simple fact that an isometry is a harmonic map. Hence the remaining open cases are the real hyperbolic spaces $H_{\mathbb{R}}^{n}$ and the complex hyperbolic spaces $H_{\mathbb{C}}^{n}$. As supporting evidence to the conjecture, we point out that Tam and the first author solved the Dirichlet problem for proper harmonic maps between real hyperbolic spaces when the boundary map is $C^{1}$ with nonvanishing energy density. In particular, if both the domain and the target are the same real hyperbolic space $H_{\mathbb{R}}^{n}$ and the boundary map is further assumed to be quasi-conformal, then it can be verified that the resulting harmonic map between $H_{\mathbb{R}}^{n}$ and itself is a rough isometry. A similar statement holds for the case of complex hyperbolic space from the work by Donnelly in [D 1].

Proposition $0.3[\mathrm{P} 1]$. Let $M$ be either a quaternionic hyperbolic space $H_{\mathbb{Q}}^{n}$ or the Cayley hyperbolic plane $H_{\mathbb{C} a}^{2}$. If $u: M \rightarrow M$ is a rough isometry, then there exists an isometry $v: M \rightarrow M$ such that $u$ is of bounded distance from $v$.

In section 2, we will prove that the uniqueness part of Conjecture 0.2 is valid. In fact, uniqueness holds in the more general setting when $M$ is a Cartan-Hadamard manifold and $N$ is only assumed to be a Hadamard space with strictly negative curvature, and both $M$ and $N$ have cocompact isometry groups. In this case, a harmonic rough isometry between $M$ and $N$ must be uniquely determined by its boundary map. Combining with Pansu's theorem [P 1], this implies that (Corollary 2.6) a harmonic rough isometry between $H_{\mathbb{Q}}^{n}$ (or $H_{\mathbb{C} a}^{2}$ ) and itself is an isometry. Recall that a geometric version of Mostow's rigidity theorem asserts that if $\Sigma_{1}$ and $\Sigma_{2}$ are two compact, rank-1 locally symmetric spaces of the same type that are homeomorphic to each other, then they are in fact isometric. By applying the Eells-Sampson theorem, if one deforms the homeomorphism to a harmonic map $u: \Sigma_{1} \rightarrow \Sigma_{2}$, then Mostow's theorem follows if we can show that the harmonic map is an isometry. Lifting $u$ to a map $\tilde{u}: M \rightarrow M$ between the universal coverings of $\Sigma_{1}$ and $\Sigma_{2}$, it follows that $\tilde{u}$ is also harmonic. The equivariant property of $\tilde{u}$ implies that it is also a rough isometry. At this point, if we can prove that $\tilde{u}$ is an isometry, then $u$ itself must be an isometry. Corollary 2.6 asserts that this is indeed the case when $M$ is either $H_{\mathbb{Q}}^{n}$ or $H_{\mathbb{C} a}^{2}$. Since we do not require the harmonic rough isometry to be equivariant for the validity of Corollary 2.6, we can view this as a generalization of Mostow's theorem for the trivial group in this setting. Using a recent result of Kleiner and Leeb [K-L], we also obtain a similar generalization to the higher rank case.

Finally, in the last section, we prove the existence part of Conjecture 0.1 for a certain class of quasi-symmetric homeomorphisms. In particular, a quasi-symmetric homeomorphism $f: S^{1} \rightarrow S^{1}$ is extendable to a quasi-conformal harmonic diffeomorphism of $H_{\mathbb{R}}^{2}$ if $f$ has isolated points which are not $C^{1}$ or have vanishing energy density, and near each of those points $f$ has an expansion of the form $r^{\alpha} h(r)$ for some positive $C^{1}$ function $h$.

The authors would like to thank Rick Schoen for suggesting us to investigate in the direction of harmonic pseudo-isometries in higher dimensions. Also, we would like to thank him for pointing out the work of Pansu. The second author would like to express his gratitude to Bernhard Leeb for sending him the reference [K-L], and also 
to Leon Simon for his interest in this work.

1. Properties of Rough-Isometries. In this section, we will prove some basic properties of rough isometries between two Hadamard spaces. Let us begin by recalling the definition of a rough isometry.

Definition 1.1. Let $X$ and $Y$ be metric spaces. A map $u: X \rightarrow Y$ is said to be a rough isometry if there exist positive constants $k, b$ and $c$, and a map $v$ from $Y$ to $X$ such that,

$$
k^{-1} d\left(x_{1}, x_{2}\right)-b \leq d\left(u\left(x_{1}\right), u\left(x_{2}\right)\right) \leq k d\left(x_{1}, x_{2}\right)+b
$$

for all $x_{1}, x_{2} \in X$, and $d(u \circ v(y), y) \leq c$ for all $y \in Y$. In this case, we say that the map $u$ is a $(k, b)$ rough isometry and that $X$ and $Y$ are rough isometrically equivalent.

It is easy to check that the map $v$ in the preceding definition is also a rough isometry from $Y$ to $X$ and $d(v \circ u(x), x) \leq c$ for all $x \in X$. Note that in general a map $u$ which satisfies (A) and maps $X$ onto $Y$ is a rough isometry from $X$ to $Y$. In fact, we can define $v: Y \rightarrow X$ by $v(y) \in u^{-1}(y)$ for $y \in Y$. Then it is easily verified that for all $y \in Y, d(u \circ v(y), y) \leq c$. In fact, we may take constant $c$ to be zero. A result of Mostow $[\mathrm{M}]$ says that a map satisfying (A) between two Cartan Hadamard manifolds of the same dimension must be onto if it is also continuous. In particular, this implies that any continuous map satisfying (A) between two such manifolds is a rough isometry. We would like to point out that if we replace the bounds of $d\left(u\left(x_{1}\right), u\left(x_{2}\right)\right)$ in the definition of rough isometry by

$$
k^{-1} d\left(x_{1}, x_{2}\right)-b \leq d\left(u\left(x_{1}\right), u\left(x_{2}\right)\right) \leq k d\left(x_{1}, x_{2}\right),
$$

then the map $u$ is said to be a pseudo-isometry. One obvious difference in the two definitions is that a rough isometry may not be continuous while a pseudo-isometry is necessarily Lipschitz.

For metric spaces, one may use triangle comparisons to define curvature bounds. This idea seems to be due to A. Wald [Wa] and it has been developed by a Russian school of mathematicians centered around A. D. Alexandrov. Let us recall the definition of a metric space with a curvature upper bound.

DEFINITION 1.2. Let $M_{k}^{2}$ be the two dimensional model space of constant curvature $k$. A complete metric space $(X, d)$ is said to have a curvature upper bound $k$, denoted by $K_{X} \leq k$, if the following two conditions are satisfied:

(1) $(X, d)$ is a length space, i.e., for any two points $P, Q \in X$, the distance $d(P, Q)$ is realized as the length of a rectifiable curve connecting $P$ and $Q$. In this case, such a distance realizing curve is called a geodesic.

(2) Let $P, Q, R$ be three points in $X$ and choices of geodesics $\gamma_{P, Q}$ of length $r$, $\gamma_{Q, R}$ of length $p$, and $\gamma_{R, P}$ of length $q$ connecting the respective points. If $k>0$ we assume that $r+p+q \leq 2 \pi / \sqrt{k}$. For any $0<\lambda<1$ let $Q_{\lambda}$ be the point on $\gamma_{Q, R}$ such that

$$
d\left(Q_{\lambda}, Q\right)=\lambda p
$$

and

$$
d\left(Q_{\lambda}, R\right)=(1-\lambda) p .
$$

Consider the possibly degenerate triangle in $M_{k}^{2}$ whose sides have length given by $p$, $q, r$ and opposite vertices $\bar{P}, \bar{Q}, \bar{R}$, there is a corresponding point $\overline{Q_{\lambda}}$ such that

$$
d\left(\overline{Q_{\lambda}}, \bar{Q}\right)=\lambda p,
$$


and

$$
d\left(\overline{Q_{\lambda}}, \bar{R}\right)=(1-\lambda) p
$$

Then $d\left(P, Q_{\lambda}\right) \leq d\left(\bar{P}, \overline{Q_{\lambda}}\right)$.

A complete metric space is said to be non-positively curved (NPC) if $K_{X} \leq 0$. Furthermore, an (NPC) space $(X, d)$ is called a Hadamard space if it is geodesically complete, namely, passing through any two points in $X$ there exists a (necessarily unique) geodesic line. By the completeness of $(X, d)$, this is equivalent to require that any geodesic of $(X, d)$ can be locally extended. Clearly, every Cartan Hadamard manifold is a Hadamard space. As a trivial infinite dimensional example, a Hilbert space is a Hadamard space.

Let $l$ be a geodesic line in an (NPC) space $(X, d)$. Then it is easy to see that for every point $p \in X$ there exists a unique point $q \in l$ such that $d(p, q)=d(p, l)$. We define a map $\pi_{l}$ from $X$ onto $l$ by $\pi_{l}(p)=q$ and call $\pi_{l}$ to be the orthogonal projection map from $X$ onto $l$. Let us first state a proposition concerning the orthogonal projection map $\pi_{l}$ which is well-known in the Riemannian manifold setting and can be proved by a standard Jacobi field argument. Here the argument is slightly more complicated and it is given in the appendix.

Proposition 1.3. Let $(X, d)$ be a metric space with curvature $K_{X} \leq-a^{2} \leq 0$. Let $L$ be a rectifiable curve in $X$ and of distance $R$ away from the geodesic line $l$. Then the orthogonal projection map $\pi_{l}$ from $X$ onto the geodesic line $l$ contracts the length of $L$ by a factor $\cosh (a R)$, i.e. $\left|\pi_{l}(L)\right| \leq \frac{|L|}{\cosh (a R)}$.

One immediately sees from the above proposition that the orthogonal projection map $\pi_{l}$ is a Lipschitz map of Lipschitz constant 1 when $X$ is an (NPC) space.

Proposition 1.4 [E]. Let $M$ and $N$ be Hadamard spaces such that either $K_{M} \leq$ $-a^{2}$ or $K_{N} \leq-a^{2}$ for some $a>0$. Let $u$ be a rough isometry between $M$ and $N$. For a geodesic segment $\beta$ with end points $m_{1}$ and $m_{2}$ in $M$, let $\delta$ be the geodesic segment connecting $u\left(m_{1}\right)$ and $u\left(m_{2}\right)$ in $N$. Then there exists a constant $c$ independent of $\beta$ such that the Hausdorff distance $d_{H}(u(\beta), \delta) \leq c$.

The following lemma readily follows from Proposition 1.4 if $N$ is locally compact. However, a Hadamard space in general is not necessarily so and some argument is needed.

LEMmA 1.5. Let $M$ and $N$ be Hadamard spaces such that the curvature of $N$ satisfies $K_{N} \leq-a^{2}$ for some $a>0$. Let $u$ be a rough isometry between $M$ and $N$. Then for any geodesic line $\beta$ in $M$, there exists a unique geodesic line $\delta$ in $N$ such that the Hausdorff distance $d_{H}(u(\beta), \delta) \leq c$, where constant $c$ is independent of $\beta$.

Proof. Let $\beta$ be a geodesic line in $M$. We parametrize $\beta$ by arclength over the interval $(-\infty, \infty)$. For each $t>0$, let $\beta_{t}$ be the geodesic segment $\left.\beta\right|_{[-t, t]}$. Let $\delta_{t}$ be the geodesic segment in $N$ determined by $u(\beta(-t))$ and $u(\beta(t))$. Then Proposition 1.4 implies that there is a constant $c$ independent of $\beta$ and $t$ such that $d_{H}\left(u\left(\beta_{t}\right), \delta_{t}\right) \leq c$. Choose a sequence of numbers $t_{i}$ such that $t_{1}>k b$ and $t_{i+1}>t_{i}+k\left(4^{i}+b+2 c\right)$. Let $\beta_{i}=\beta_{t_{i}}$ and $\delta_{i}=\delta_{t_{i}}$. We claim that the sequence of geodesic segments $\delta_{i}$ converges to a geodesic line $\delta$. Then it is evident that $d_{H}(u(\beta), \delta) \leq c$. Note that for $0<i<j$,

$$
\begin{aligned}
\sup _{x \in \delta_{i}} d\left(x, \delta_{j}\right) & \leq \sup _{x \in \delta_{i}} d\left(x, u\left(\beta_{j}\right)\right)+d_{H}\left(u\left(\beta_{j}\right), \delta_{j}\right) \\
& \leq \sup _{x \in \delta_{i}} d\left(x, u\left(\beta_{i}\right)\right)+d_{H}\left(u\left(\beta_{j}\right), \delta_{j}\right) \\
& \leq d_{H}\left(\delta_{i}, u\left(\beta_{i}\right)\right)+d_{H}\left(u\left(\beta_{j}\right), \delta_{j}\right) \\
& \leq 2 c .
\end{aligned}
$$


Let $l_{i}$ be the geodesic line containing $\delta_{i}$. Then we have $\pi_{l_{i+1}}\left(\delta_{i}\right) \subset \delta_{i+1}$. In fact,

$$
\begin{aligned}
d\left(u\left(\beta\left(t_{i}\right)\right), u\left(\beta\left(t_{i+1}\right)\right)\right) & \geq k^{-1} d\left(\beta\left(t_{i}\right), \beta\left(t_{i+1}\right)\right)-b \\
& \geq k^{-1}\left(t_{i+1}-t_{i}\right)-b \\
& >2 c
\end{aligned}
$$

and

$$
\begin{aligned}
d\left(u\left(\beta\left(t_{i}\right)\right), u\left(\beta\left(-t_{i+1}\right)\right)\right) & \geq k^{-1} d\left(\beta\left(t_{i}\right), \beta\left(-t_{i+1}\right)\right)-b \\
& \geq k^{-1}\left(t_{i+1}+t_{i}\right)-b>2 c
\end{aligned}
$$

In view of (1.1), (1.2) and (1.3), one concludes that $\pi_{l_{i+1}}\left(u\left(\beta\left(t_{i}\right)\right)\right) \in \delta_{i+1}$. Similarly, $\pi_{l_{i+1}}\left(u\left(\beta\left(-t_{i}\right)\right)\right) \in \delta_{i+1}$. Thus, $\pi_{l_{i+1}}\left(\delta_{i}\right) \subset \delta_{i+1}$. Now pick up a point $x_{1} \in \delta_{1}$ and define a sequence of points $x_{i} \in \delta_{i}$ inductively by $x_{i+1}=\pi_{l_{i+1}}\left(x_{i}\right)$. We first show that the sequence of points $x_{i}$ converges to some point $x \in N$. For any $i \geq 1$, let $\gamma_{i}$ be the segment of $\delta_{i}$ joining $x_{i}$ to $u\left(\beta\left(t_{i}\right)\right)$. Let $L_{i}=\left|\gamma_{i}\right|$ be the length of $\gamma_{i}$. In view of (1.1) and the choice of $t_{i}$,

$$
\begin{aligned}
L_{i} & =d\left(x_{i}, u\left(\beta\left(t_{i}\right)\right)\right) \geq d\left(\pi_{l_{i}}\left(u\left(\beta\left(t_{i-1}\right)\right)\right), u\left(\beta\left(t_{i}\right)\right)\right. \\
& \geq d\left(u\left(\beta\left(t_{i-1}\right)\right), u\left(\beta\left(t_{i}\right)\right)\right)-2 c \\
& \geq k^{-1}\left(t_{i}-t_{i-1}\right)-b-2 c \\
& \geq 4^{i-1} .
\end{aligned}
$$

Denote the length of $\pi_{l_{i+1}}\left(\gamma_{i}\right)$ by $H_{i}$. Then by (1.1),

$$
\begin{aligned}
H_{i} & \geq L_{i}-d\left(x_{i}, l_{i+1}\right)-d\left(u\left(\beta\left(t_{i}\right)\right), l_{i+1}\right) \\
& \geq L_{i}-4 c .
\end{aligned}
$$

Let $R_{i}$ be the distance between $\gamma_{i}$ and $l_{i+1}$. Proposition 1.3 then implies that

$$
H_{i} \cosh \left(a R_{i}\right) \leq L_{i}
$$

Using (1.4) and (1.5), we obtain from (1.6) that

$$
R_{i} \leq \frac{1}{a}\left(\frac{8 c}{L_{i}-4 c}+\sqrt{\frac{8 c}{L_{i}-4 c}}\right) \leq c_{1} 2^{-i}
$$

for some constant $c_{1}$ depending on $a$ and $c$ only. Thus, for a given $i$, there exists a point $y \in \delta_{i}$ between $x_{i}$ and $u\left(\beta\left(t_{i}\right)\right)$ such that $d\left(y, l_{i+1}\right) \leq c_{1} 2^{-i}$. The same argument shows that there exists a point $z \in \delta_{i}$ between $x_{i}$ and $u\left(\beta\left(-t_{i}\right)\right)$ such that $d\left(z, l_{i+1}\right) \leq c_{1} 2^{-i}$. The convexity of the function $d\left(x, l_{i+1}\right)$ then implies that

$$
d\left(x_{i}, x_{i+1}\right)=d\left(x_{i}, l_{i+1}\right) \leq c_{1} 2^{-i} .
$$

This implies that $x_{i}$ is a Cauchy sequence and by the completeness of $N, x_{i}$ must converge to some point $x \in N$.

To show the convergence of $\delta_{i}$, we parametrize $\delta_{i}$ by arclength over an interval $\left[-b_{i}, a_{i}\right]$ containing 0 such that $\delta_{i}(0)=x_{i}$. By (1.4), one sees that both $a_{i}$ and $b_{i}$ goes to infinity. For each fixed real number $s$, we will show that $\lim _{i \rightarrow \infty} \delta_{i}(s)$ exists in $N$. In the following, we give the argument for the case that $s>0$. For any $i \geq 1$, by (1.1), there exists a point $P \in \delta_{i+1}$ such that

$$
d\left(u\left(\beta\left(t_{i}\right)\right), P\right)=d\left(u\left(\beta\left(t_{i}\right)\right), l_{i+1}\right) \leq 2 c .
$$


Consider the ordered sequence of points $x_{i}, u\left(\beta\left(t_{i}\right)\right), P$ and $x_{i+1}$. Since the curvature $K_{N} \leq-a^{2}, N$ in particular is an (NPC) space. By the quadrilateral comparison theorem (see [K-S] or the appendix), there exists a convex Euclidean quadrilateral $A B C D$ in $\mathbb{R}^{2}$ satisfying $|A B|=d\left(x_{i}, u\left(\beta\left(t_{i}\right)\right)\right)=L_{i},|B C|=d\left(u\left(\beta\left(t_{i}\right)\right), P\right),|C D|=$ $d\left(P, x_{i+1}\right)$ and $|D A|=d\left(x_{i+1}, x_{i}\right)$ such that if we let $E$ and $F$ be the points on the sides $A B$ and $C D$ respectively with $|A E|=\lambda$ and $|D F|=\mu$, then $d\left(\delta_{i}(\lambda), \delta_{i+1}(\mu)\right) \leq|E F|$. In particular, that shows the angles $\angle A D C \geq \pi / 2$ and $\angle B C D \geq \pi / 2$. Choose points $G$ and $J$ on the sides $A B$ and $C D$, respectively, such that $|J D|=s$ and that $G J$ is perpendicular to $C D$. Let $H$ be the projection point of $A$ onto the line passing through $C$ and $D$. Then

$$
\begin{aligned}
|H J| & =|H D|+|D J| \leq|A D|+s \\
& \leq c_{1} 2^{-i}+s .
\end{aligned}
$$

Let $K$ be the point on $A B$ such that $K C$ is perpendicular to $C D$. In case $\angle A B C>\pi / 2$, we see that $|A H|>|G J|$. In this case, we conclude that

$$
|G J|<|A H| \leq|A D| \leq c_{1} 2^{-i}
$$

If $\angle A B C \leq \pi / 2$, then $|K C| \leq|B C|$. Let us then consider the quadrilateral $A K C H$. Since $A H, G J$, and $K C$ are all perpendicular to $H C$, we conclude that

$$
\begin{aligned}
|G J| & =\frac{|A H||J C|}{|H C|}+\frac{|K C||H J|}{|H C|} \\
& \leq|A D|+\frac{|B C||H J|}{|C D|} \\
& \leq c_{1} 2^{-1}+\frac{(2 c)\left(c_{1} 2^{-1}+s\right)}{|C D|}
\end{aligned}
$$

where we have used (1.7) and (1.8). However, since

$$
\begin{aligned}
|C D| & \geq|A B|-|A D|-|B C| \\
& \geq 4^{i-1}-c_{1} 2^{-i}-2 c
\end{aligned}
$$

this asserts that

$$
|G J| \leq c(s) 2^{-i}
$$

for some constant $c(s)$ depending only on $s$. Finally, let $T$ be a point on the side $A B$ such that $|A T|=s$. Then using (1.7) and (1.9), we obtain

$$
\begin{aligned}
d\left(\delta_{i}(s), \delta_{i+1}(s)\right) & \leq|T J| \\
& \leq|G J|+|G T| \\
& \leq|G J|+|| G A|-| A T|| \\
& =|G J|+|| G A|-| J D|| \\
& \leq|G J|+|A D|+|G J| \\
& \leq c(s) 2^{-i}
\end{aligned}
$$

It is now clear that (1.10) implies that the $\lim _{i \rightarrow \infty} \delta_{i}(s)$ exists.

To show the uniqueness of such geodesic line $\delta$, let $\delta_{1}$ and $\delta_{2}$ be two geodesic lines parametrized by arc-length such that $d_{H}\left(u(\beta), \delta_{i}\right) \leq c$ for $i=1$ and 2 . This implies that $d_{H}\left(\delta_{1}, \delta_{2}\right) \leq 2 c$. Using Proposition 1.3, one shows that for any $t_{1}$ and 
$t_{2}, d\left(\left.\delta_{1}\right|_{\left[t_{1}, t_{2}\right]}, \delta_{2}\right) \rightarrow 0$ as $t_{2}-t_{1} \rightarrow \infty$. In particular, $\liminf \inf _{t \rightarrow \infty} d\left(\delta_{1}(t), \delta_{2}\right)=0$ and $\liminf \operatorname{in}_{t \rightarrow-\infty} d\left(\delta_{1}(t), \delta_{2}\right)=0$. Convexity of the function $d\left(x, \delta_{2}\right)$ now implies that $d\left(\delta_{1}(t), \delta_{2}\right)=0$ for all $t$. Hence, $\delta_{1}=\delta_{2}$, and the proof is complete.

In the following, we will use Lemma 1.5 to discuss the behavior of a rough isometry at the infinity. For an (NPC) space, one defines its geometric boundary as in the case of Cartan Hadamard manifolds. Let $X$ be an (NPC) space. Two geodesic rays $\beta_{1}$ and $\beta_{2}$ of $X$ are defined to be equivalent if their Hausdorff distance $d_{H}\left(\beta_{1}, \beta_{2}\right)$ is finite. Then the geometric boundary of $X$ is the set of equivalence classes of geodesic rays and it is denoted by $\partial_{\infty} X$. The geometric boundary $\partial_{\infty} X$ is also referred to be the sphere at infinity for the space $X$. It is clear that for $x \in X$ and $p \in \partial_{\infty} X$, there exists a unique geodesic ray emanating from $x$ which represents $p$. Thus, the pointed Hausdorff topology on rays emanating from $x \in X$ induces a topology on $\partial_{\infty} X$. It is easy to see that this topology is independent of the base point $x$. In the following, it is assumed that $\partial_{\infty} X$ carries such a topology. When $(X, d)$ is a Hadamard space, one checks that $\partial_{\infty} X$ is homeomorphic to the unit sphere centered at an arbitrary point $x \in X$. Let $u, M$ and $N$ be the same as in Proposition 1.4. Applying Lemma 1.5, one concludes that for a geodesic ray $\beta$ emanating from $x$ in $M$, there exists a geodesic ray $\delta$ in $N$ emanating from $u(x)$ such that $d_{H}(u(\beta), \delta) \leq c$, where $c$ is a constant independent of $\beta$.

Proposition 1.6. Let $M$ and $N$ be Hadamard spaces. Suppose that either $K_{M} \leq-a^{2}$ or $K_{N} \leq-a^{2}$ for some $a>0$. Then every rough isometry $u: M \rightarrow N$ induces a homeomorphism between the spheres at the infinity. Moreover, two such rough isometries $u_{1}$ and $u_{2}$ between $M$ and $N$ induce the same boundary map at the infinity if and only if $d\left(u_{1}(x), u_{2}(x)\right) \leq c$ for all $x \in M$.

Proof. Fix a point $x$ in $M$. For $p \in \partial_{\infty} M$, let $\beta$ be the geodesic ray emanating from $x$ which represents $p$. By the remark following Lemma 1.5, there exists a geodesic ray $\delta$ emanating from $u(x)$ such that $d_{H}(u(\beta), \delta) \leq c$, where $c$ is independent of $\beta$. Let $q \in \partial_{\infty} N$ be the point represented by the ray $\delta$. Then $\phi(p)=q$ defines a map from $\partial_{\infty} M$ to $\partial_{\infty} N$. It is easy to see that $\phi$ is a surjective homeomorphism.

To prove the second conclusion, note that it is clear that $u_{1}$ and $u_{2}$ induce the same boundary map if $d\left(u_{1}, u_{2}\right) \leq c$ for some constant $c$. Conversely, suppose $u_{1}$ and $u_{2}$ induce the same boundary map at infinity. We first assume that $K_{N} \leq-a^{2}$ for some $a>0$. This assumption asserts that $N$ has more than one geodesic line. In particular, $\partial_{\infty} N$ has more than two points. Thus, by the surjectivity of the induced boundary map of $u_{1}$, we conclude that $\partial_{\infty} M$ contains more than two points. Therefore, for every $p \in M$, there exists a geodesic line $l_{1}$ in $M$ passing through $p$ and a point $x \in M$ such that $x$ is not on the line $l_{1}$. We parametrize $l_{1}$ over $(-\infty, \infty)$ such that $l_{1}(0)=p$. Let $L$ be the broken geodesic connecting $l_{1}(-1), x$ and $l_{1}(1)$. By the continuity of the orthogonal projection map $\pi_{l_{1}}$, one concludes that $\pi_{l_{1}}(L)$ is connected and hence must contain point $p$. In particular, one finds a point $y \in L$ such that $\pi_{l_{1}}(y)=p$. It is clear that $y$ is not on the line $l_{1}$. Let $l_{2}$ be the geodesic line passing through $p$ and $y$. It is then easy to see that $\pi_{l_{1}}\left(l_{2}\right)=p$ as $M$ is an (NPC) space. By Lemma 1.5, there exist geodesic lines $\gamma_{1}$ and $\gamma_{2}$ in $N$ such that $d_{H}\left(u_{1}\left(l_{i}\right), \gamma_{i}\right) \leq c$ and $d_{H}\left(u_{2}\left(l_{i}\right), \gamma_{i}\right) \leq c$ for $i=1$, 2. In particular, we have $u_{1}(p) \in T_{c} \gamma_{1} \cap T_{c} \gamma_{2}$ and $u_{2}(p) \in T_{c} \gamma_{1} \cap T_{c} \gamma_{2}$, where $T_{c} \gamma_{i}$ is the tubular neighborhood of $\gamma_{i}$ of size $c, i=1$ and 2. Let $v_{1}$ and $v_{2}$ be two rough isometries from $N$ into $M$ such that $d\left(u_{i} \circ v_{i}(w), w\right) \leq f$ and $d\left(v_{i} \circ u_{i}(z), z\right) \leq f$ for all $z \in M$ and $w \in N$, where $f$ is a constant. Without loss of generality, we may assume that $u_{i}$ and $v_{i}$ are $(k, b)$ rough isometries. For $z=v_{1}(w) \in v_{1}\left(T_{c} \gamma_{1}\right)$, it is easy 
to see that

$$
\begin{aligned}
d\left(z, l_{1}\right) & =d\left(v_{1}(w), l_{1}\right) \\
& \leq k d\left(u_{1} \circ v_{1}(w), u_{1}\left(l_{1}\right)\right)+b \\
& \leq k d\left(u_{1} \circ v_{1}(w), w\right)+k d\left(w, \gamma_{1}\right)+k d_{H}\left(u_{1}\left(l_{1}\right), \gamma_{1}\right)+b \\
& \leq k f+2 k c+b .
\end{aligned}
$$

Thus, we conclude that $v_{1}\left(T_{c} \gamma_{1}\right) \subset T_{\tilde{c}} l_{1}$, where $\tilde{c}=k f+2 k c+b$. Similarly, we get $v_{1}\left(T_{c} \gamma_{2}\right) \subset T_{\tilde{c}} l_{2}$. Since $u_{2}(p) \in T_{c} \gamma_{1} \cap T_{c} \gamma_{2}$, this implies that $v_{1}\left(u_{2}(p)\right) \in T_{\tilde{c}} l_{1} \cap T_{\tilde{c}} l_{2}$. Let us set $q=v_{1}\left(u_{2}(p)\right)$. If we let $q_{1}$ to be the point $\pi_{l_{1}}(q)$ and $q_{2}$ the point $\pi_{l_{2}}(q)$, then noting that $\pi_{l_{1}}\left(l_{2}\right)=p$ we obtain

$$
\begin{aligned}
d\left(u_{1}(p), u_{2}(p)\right) & \leq d\left(u_{1}(p), u_{1}(q)\right)+d\left(u_{1}(q), u_{2}(p)\right) \\
& \leq k d(p, q)+b+f \\
& \leq k d\left(p, q_{1}\right)+k d\left(q, q_{1}\right)+b+f \\
& =k d\left(\pi_{l_{1}}\left(q_{2}\right), \pi_{l_{1}}(q)\right)+k d\left(q, l_{1}\right)+b+f \\
& \leq k d\left(q_{2}, q\right)+k \tilde{c}+b+f \\
& =k d\left(q, l_{2}\right)+k \tilde{c}+b+f \\
& \leq 2 k \tilde{c}+b+f
\end{aligned}
$$

where we have used the fact that $M$ is an (NPC) space and the projection $\pi_{l_{1}}$ is distance decreasing. This completes the proof for the case $K_{N} \leq-a^{2}$ for some $a>0$. Now if $K_{M} \leq-a^{2}$ for some $a>0$, then the preceding argument can be applied to show that $d\left(v_{1}(y), v_{2}(y)\right) \leq c$ for all $y \in N$. Thus, for $x \in M$, we conclude that

$$
\begin{aligned}
d\left(u_{1}(x), u_{2}(x)\right) & \leq k d\left(v_{1}\left(u_{1}(x)\right), v_{1}\left(u_{2}(x)\right)\right)+b \\
& \leq k d\left(x, v_{1}\left(u_{2}(x)\right)\right)+k f+b \\
& \leq k d\left(x, v_{2}\left(u_{2}(x)\right)\right)+k d\left(v_{2}\left(u_{2}(x)\right), v_{1}\left(u_{2}(x)\right)\right)+k f+k b \\
& \leq k f+k c+k f+k b,
\end{aligned}
$$

and the proof is complete.

In $[\mathrm{M}]$, Mostow considered the notion of quasi-conformal mappings over a division algebra $K$ and showed that a pseudo-isometry between a rank-1 symmetric space and itself that is equivariant with respect to some cocompact group actions must induce a boundary homeomorphism which is also quasi-conformal over the division algebra $K$ associated with the symmetric space. This conclusion is also valid for rough isometries.

Corollary 1.7 [M]. Let $M$ be a rank-1 symmetric space. If $u: M \rightarrow M$ is a rough isometry, then $u$ induces a quasi-conformal homeomorphism on the geometric boundary of $M$.

To justify our claim that Conjecture 0.2 is a generalization of Conjecture 0.1 , we will show that these two conjectures are equivalent when $M=N=H_{\mathbb{R}}^{2}$.

THEOREM 1.8. Let $u$ be a harmonic map between the real hyperbolic plane $H_{\mathbb{R}}^{2}$ and itself. Then $u$ is a quasi-conformal diffeomorphism if and only if $u$ is a rough isometry.

Proof. First observe that a harmonic rough isometry is a pseudo-isometry, hence it will be automatically surjective. Let $u$ be a $(k, b)$ rough isometry. Since $u$ is continuous, for every $p \in H_{\mathbb{R}}^{2}$ and $r>3 k^{2} b$, we have $B_{u(p)}(r / k) \subset u\left(B_{p}(r)\right.$ ) (see [M, p. 74]). By [J], we know then the Jacobian of $u$ is positive at $p$. Thus, the Jacobian of $u$ is positive everywhere. Since the boundary map induced by $u$ is a homeomorphism, 
we conclude that $u$ is a diffeomorphism of $H_{\mathbb{R}}^{2}$ and itself. On the other hand, since $u\left(B_{p}(b)\right) \subset B_{u(p)}((k+1) b)$, the gradient estimate of Cheng [C] implies that the energy density of $u$ is uniformly bounded above by some constant. Now a result of Wan [W] says that $u$ must be a quasi-conformal diffeomorphism. Conversely, if $u$ is a quasiconformal harmonic diffeomorphism, then the same theorem of Wan [W] also asserts that $u$ has energy density uniformly bounded from above and below by two constants $c_{1}, c_{2}>0$ such that

$$
c_{1} \leq e(u) \leq c_{2}
$$

The upper bound implies that

$$
d(u(x), u(y)) \leq c_{2} d(x, y) .
$$

The lower bound together with the assumption that $u$ is quasi-conformal implies that the differential of $u$ is uniformly bounded away from 0 . This gives the estimate in the opposite direction, and hence $u$ is a rough isometry.

2. Uniqueness. In this section, we will prove the uniqueness part of Conjecture 0.2. In fact, uniqueness holds for general Cartan-Hadamard manifolds with cocompact isometry groups. Specifically, we will show that a harmonic rough isometry is uniquely determined by its induced boundary map at infinity. Together with Proposition 0.3 , we are able to conclude that a harmonic rough isometry between a quaternionic hyperbolic space ( or Cayley hyperbolic plane) and itself must be an isometry. Notice that we do not require the harmonic map to be equivariant with respect to any group action. In particular, this can be viewed as a generalization of the Mostow rigidity theorem to the universal coverings.

In the following, we shall consider more generally harmonic maps from smooth manifolds into metric spaces. The fundamental theory of this setting has been introduced and studied by Korevaar and Schoen in their important paper [K-S] . We shall show that the aforementioned uniqueness result is also valid for such harmonic maps. Let us begin with the following two lemmas which are also of independent interest. The idea of their proofs was originated in forthcoming paper of Korevaar and Schoen, where they proved that the distance between two harmonic maps is subharmonic, however more technical effort is required to deal with the uniqueness situation.

Lemma 2.1. Let $M$ be a Riemannian manifold and $N$ an (NPC) space. Let $u$ be a harmonic map from $M$ to $N$. Then $\Delta d^{2}(u(x), p) \geq 2 e(u)(x)$ weakly, where $p$ is a fixed point in $N$ and $e(u)(x)$ is the energy density function of $u$.

Proof. Let $\eta(x)$ be a nonnegative smooth function with compact support in $M$. Let $\Omega$ be a compact domain containing the support of $\eta$. For $0 \leq t \leq 1$, let $u_{t}$ be the map such that for each $x \in M, u_{t}(x)$ is on the geodesic segment joining $u(x)$ to $p$ with

$$
d\left(u_{t}(x), u(x)\right)=t \eta(x) d(u(x), p) .
$$

For $x, y \in M$, consider the ordered sequence of points $p, u(x)$ and $u(y)$. Since $N$ is an (NPC) space, there exists a comparison triangle with corresponding vertices $P, Q$ and $R$ in $\mathbb{R}^{2}$ such that $d(p, u(x))=|P-Q|, d(u(x), u(y))=|Q-R|$ and $d(u(y), p)=|R-P|$. Let $A$ and $B$ be the points on the side $P R$ and $P Q$ respectively such that $|R-A|=d\left(u_{t}(y), u(y)\right)$ and $|Q-B|=d\left(u_{t}(x), u(x)\right)$. Then we have

$$
d\left(u_{t}(x), u_{t}(y)\right) \leq|A-B|
$$


Direct computation yields

$$
\begin{aligned}
|A-B|^{2}=|R-Q|^{2}-t(\eta(x)+\eta(y))|R-Q|^{2} \\
\quad+t(\eta(y)-\eta(x))\left(|P-Q|^{2}-|P-R|^{2}\right)+O\left(t^{2}\right)
\end{aligned}
$$

Therefore,

$$
\begin{aligned}
d^{2}\left(u_{t}(y), u_{t}(x)\right) \leq d^{2}(u(y), u(x))-t(\eta(x)+\eta(y)) d^{2}(u(y), u(x)) & \\
& +t(\eta(y)-\eta(x))\left(d^{2}(u(x), p)-d^{2}(u(y), p)\right)+O\left(t^{2}\right)
\end{aligned}
$$

Integrating and averaging on the subset $|x-y| \leq \epsilon$ of $\Omega \times \Omega$ we deduce by letting $\epsilon \rightarrow 0$ that

$$
\begin{aligned}
E_{\Omega}\left(u_{t}\right) \leq E_{\Omega}(u)-2 t \int_{\Omega} \eta(x) e(u)(x) d x \\
\quad-t \int_{\Omega}\left\langle\nabla \eta(x), \nabla d^{2}(u(x), p)\right\rangle d x+O\left(t^{2}\right)
\end{aligned}
$$

Since $u$ is harmonic and $u_{t}=u$ on $\partial \Omega$, we have

$$
E_{\Omega}(u) \leq E_{\Omega}\left(u_{t}\right)
$$

Hence we conclude that

$$
2 \int_{\Omega} \eta(x) e(u)(x) d x \leq-\int_{\Omega}\left\langle\nabla \eta(x), \nabla d^{2}(u(x), p)\right\rangle d x+O(t) .
$$

After letting $t \rightarrow 0$, we derived the inequality

$$
2 \int_{M} \eta(x) e(u)(x) d x \leq-\int_{M}\left\langle\nabla \eta(x), \nabla d^{2}(u(x), p)\right\rangle d x
$$

and the lemma is proved.

LEMMA 2.2. Let $M$ be a complete Riemannian manifold and $N$ a complete metric space with $K_{N}<0$. Let $u$ and $v$ be two harmonic maps from $M$ to $N$ such that $d(u, v)$ is constant. Then either $u \equiv v$ or both $u$ and $v$ have their image lie on a geodesic line.

Proof. Suppose that $u \neq v$. For each $0<t<1$, define map $u_{t}$ such that $u_{t}(x)$ is the point on the geodesic segment joining $u(x)$ and $v(x)$ with

$$
d\left(u_{t}(x), u(x)\right)=t d(u(x), v(x)) .
$$

We claim that $u_{t}$ is a harmonic map from $M$ into $N$. In fact, for any compact smooth domain $\Omega \subset M$, let $w_{t}$ be the harmonic map from $\Omega$ to $N$ such that $w_{t}=u_{t}$ on $\partial \Omega$. Such $w_{t}$ exists by the fact that $N$ has negative curvature, which in particular is an (NPC) space (see $[\mathrm{K}-\mathrm{S}])$. From the argument in $[\mathrm{K}-\mathrm{S}]$, we have

$$
\Delta d\left(w_{t}, u\right) \geq 0, \quad \Delta d\left(w_{t}, v\right) \geq 0 .
$$

Hence, the maximum principle implies that

$$
\begin{aligned}
d^{2}\left(w_{t}(x), u(x)\right) & \leq \sup _{y \in \partial \Omega} d^{2}\left(w_{t}(y), u(y)\right) \\
& =\sup _{y \in \partial \Omega} d^{2}\left(u_{t}(y), u(y)\right) \\
& =t^{2} d^{2}(u(x), v(x))
\end{aligned}
$$

for $x \in \Omega$. Thus,

$$
d\left(w_{t}(x), u(x)\right) \leq t d(u(x), v(x))
$$


and similarly,

$$
d\left(w_{t}(x), v(x)\right) \leq(1-t) d(u(x), v(x)) .
$$

Triangle inequality implies that

$$
d\left(w_{t}(x), u(x)\right)+d\left(w_{t}(x), v(x)\right)=d(u(x), v(x)) .
$$

Therefore, $w_{t}(x)$ lies on the geodesic segment joining $u(x)$ and $v(x)$ and

$$
d\left(w_{t}(x), u(x)\right)=t d(u(x), v(x)) .
$$

The definition of $u_{t}$ implies that $u_{t}=w_{t}$ on $\Omega$. In particular, $u_{t}$ must be harmonic, and the claim follows.

We will now show that

$$
d(u(x), u(y))=d\left(u_{t}(x), u_{t}(y)\right)
$$

for all $x$ and $y$ in $M$. More generally, we will argue that if $f$ and $g$ are two harmonic maps from $M$ into $N$ with $d(f, g)$ being constant, then $d(f(y), f(x))=d(g(y), g(x))$ for all $x$ and $y$. Let $\eta(x)$ be a nonnegative smooth function with compact support in $M$. Let $\Omega$ be a compact domain containing the support of $\eta$. For $0 \leq t \leq 1$, let $f_{t}$ and $g_{t}$ be the maps such that for each $x \in M, f_{t}(x)$ and $g_{t}(x)$ are on the geodesic segment joining $f(x)$ and $g(x)$ with

$$
d\left(f_{t}(x), f(x)\right)=t \eta(x) d(f(x), g(x))
$$

and

$$
d\left(g_{t}(x), g(x)\right)=t \eta(x) d(f(x), g(x)) .
$$

For $x$ and $y$ in $M$, consider the ordered sequence of four points $f(y), f(x), g(x)$ and $g(y)$. Since $N$ is an (NPC) space, there exists a comparison convex quadrilateral with corresponding vertices $P, Q, R$ and $S$ in $\mathbb{R}^{2}$ such that $d(f(y), f(x))=|P-Q|$, $d(f(x), g(x))=|Q-R|, d(g(x), g(y))=|R-S|$ and $d(g(y), f(y))=|S-P|$. Let $P_{t}$ and $S_{t}$ be the points on the side $P S$ such that $\left|P-P_{t}\right|=d\left(f_{t}(y), f(y)\right)$ and $\left|S-S_{t}\right|=d\left(g(y), g_{t}(y)\right)$. Similarly, define $Q_{t}$ and $R_{t}$ to be the points on the side $Q R$ such that $\left|Q-Q_{t}\right|=d\left(f(x), f_{t}(x)\right)$ and $\left|R-R_{t}\right|=d\left(g(x), g_{t}(x)\right)$. Then we have

$$
d\left(f_{t}(x), f_{t}(y)\right) \leq\left|P_{t}-Q_{t}\right|
$$

and

$$
d\left(g_{t}(x), g_{t}(y)\right) \leq\left|S_{t}-R_{t}\right|
$$

Using the fact that $|P-S|=|Q-R|$, a direct computation shows that

$$
\begin{aligned}
\mid P_{t}- & \left.Q_{t}\right|^{2}+\left|S_{t}-R_{t}\right|^{2} \\
& =|P-Q|^{2}+|S-R|^{2}-t(\eta(x)+\eta(y))|Q-P+S-R|^{2} \\
& \quad+2 t^{2}|(\eta(x)-\eta(y))(Q-R)+\eta(y)(Q-P)+\eta(y)(S-R)|^{2} \\
& \leq|P-Q|^{2}+|S-R|^{2}-t(\eta(x)+\eta(y))(|Q-P|-|S-R|)^{2} \\
& \quad+4 t^{2}\left[(\eta(x)-\eta(y))^{2}|Q-R|^{2}+2 \eta^{2}(y)|Q-P|^{2}+2 \eta^{2}(y)|S-R|^{2}\right] .
\end{aligned}
$$


Hence, the quadrilateral comparison asserts that

$$
\begin{aligned}
& d^{2}\left(f_{t}(x), f_{t}(y)\right)+d^{2}\left(g_{t}(x), g_{t}(y)\right) \\
& \leq d^{2}(f(x), f(y))+d^{2}(g(x), g(y)) \\
& -t(\eta(x)+\eta(y))(d(f(x), f(y))-d(g(x), g(y)))^{2} \\
& +4 t^{2}(\eta(x)-\eta(y))^{2} d^{2}(f(x), g(x)) \\
& +8 t^{2}\left(\eta^{2}(y) d^{2}(f(x), f(y))+\eta^{2}(y) d(g(x), g(y))^{2}\right) .
\end{aligned}
$$

For a fix point $x$ and a tangent vector $Z$ at $x$, let us denote $\exp _{x}(s Z)$ to be the geodesic emanating from $x$ in the direction $Z$. Using a regularity theorem of [K-S], the harmonic maps $f$ and $g$ are Lipschitz continuous. Hence, the functions $d\left(f(x), f\left(\exp _{x}(s Z)\right)\right)$ and $d\left(g(x), g\left(\exp _{x}(s Z)\right)\right)$ are differentiable almost everywhere. Let us denote the derivatives with respect to $s$ evaluated at $s=0$ by $\left|f_{*}(Z)\right|$ and $\left|g_{*}(Z)\right|$, respectively. Integrating and averaging on the subset $|x-y| \leq \epsilon$ of $\Omega \times \Omega$ and letting $\epsilon \rightarrow 0$, we deduce that

$$
\begin{aligned}
& E_{\Omega}\left(f_{t}\right)+E_{\Omega}\left(g_{t}\right) \\
& \leq E_{\Omega}(f)+E_{\Omega}(g) \\
& \quad-2 t \int_{S \Omega} \eta(x)\left(\left|f_{*}(Z)\right|-\left|g_{*}(Z)\right|\right)^{2} d \mu(x, Z)+c t^{2},
\end{aligned}
$$

where $S \Omega$ is the unit tangent bundle over $\Omega$ and $c$ is a constant independent of $t$. Since both $f$ and $g$ are harmonic and $f_{t}=f, g_{t}=g$ on $\partial \Omega$, we have

$$
\begin{aligned}
& E_{\Omega}(f) \leq E_{\Omega}\left(f_{t}\right), \\
& E_{\Omega}(g) \leq E_{\Omega}\left(g_{t}\right) .
\end{aligned}
$$

In particular, we conclude that

$$
\int_{S \Omega} \eta(x)\left(\left|f_{*}(Z)\right|-\left|g_{*}(Z)\right|\right)^{2} d \mu(x, Z) \leq c t .
$$

Letting $t \rightarrow 0$, we have

$$
\int_{S \Omega} \eta(x)\left(\left|f_{*}(Z)\right|-\left|g_{*}(Z)\right|\right)^{2} d \mu(x, Z)=0 .
$$

Since $\eta(x)$ is arbitrary, we conclude that $\left|f_{*}(Z)\right|=\left|g_{*}(Z)\right|$ for almost all tangent vector $Z$. Clearly, this implies that $d(f(y), f(x))=d(g(y), g(x))$ for all $x$ and $y$. In particular, we have $d(u(x), u(y))=d\left(u_{t}(x), u_{t}(y)\right)$ for all $x, y$ and $0 \leq t \leq 1$.

Fix any point $x \in M$ and let $\gamma$ be the geodesic line containing $u(x)$ and $v(x)$. If the image of $u$ is not on $\gamma$, then there exists $y$ such that $u(y)$ is not on $\gamma$. Consider the ordered sequence of four points $u(y), u(x), v(x)$ and $v(y)$. Since $N$ is an (NPC) space, there exists a comparison convex quadrilateral with corresponding vertices $A$, $B, C$ and $D$ in $\mathbb{R}^{2}$ such that $d(u(y), u(x))=|A-B|, d(u(x), v(x))=|B-C|$, $d(v(x), v(y))=|C-D|$ and $d(v(y), u(y))=|D-A|$. Let $A_{t}$ and $B_{t}$ be the points on the side $A D$ and $B C$ respectively such that $\left|A-A_{t}\right|=t|A-D|$ and $\left|B-B_{t}\right|=t|B-C|$, then

$$
\begin{aligned}
\left|A_{t}-B_{t}\right| & =|A-B| \\
& =d(u(y), u(x)) \\
& =d\left(u_{t}(y), u_{t}(x)\right) .
\end{aligned}
$$


Therefore, the quadrilateral $A B C D$ can be isometrically embedded in the space $N$. This contradicts the assumption that $K_{N}<0$, and the lemma follows.

THEOREM 2.3. Let $M$ be a Cartan Hadamard manifold and $N$ a Hadamard space with $K_{N} \leq-a^{2}<0$. Suppose that both $M$ and $N$ have cocompact isometry groups. If $u$ and $v$ are two harmonic rough isometries from $M$ to $N$ which induce the same boundary map at the infinity, then $u \equiv v$.

Proof. Let us first observe that since both $u$ and $v$ are rough isometries from $M$ to $N$, Proposition 1.6 applies and $u$ and $v$ indeed induce boundary maps at the infinity. In fact, Proposition 1.6 shows that both $u$ and $v$ induce a homeomorphism between the spheres at infinity of $M$ and $N$. Moreover, the assumption that they share the same boundary map implies that $d(u, v) \leq c$. In particular, the sphere at infinity $\partial_{\infty} N$ of $N$ is homeomorphic to $S^{n-1}$, where $n=\operatorname{dim} M$, which is evidently compact. By the fact that $N$ is a Hadamard space, one easily verifies that the spheres of $N$ are homeomorphic to $\partial_{\infty} N$. This asserts that $N$ is locally compact. Harmonicity also implies that both $u$ and $v$ are uniformly Lipschitz continuous on $M$. Indeed, since $M$ has cocompact isometry group, there exists a compact subset $A$ in $M$ such that for every point $x \in M$ there exists an isometry $\phi$ on $M$ with $\phi(x) \in A$. Choose $R>0$ and point $p \in A$ such that $A \subset B_{p}(R)$. For $x \in M$, choose an isometry $\phi$ on $M$ such that $\phi(x) \in A$. Then it is clear that

$$
\phi\left(B_{x}(2 R)\right)=B_{\phi(x)}(2 R) \subset B_{p}(4 R) .
$$

Note that the map $u \circ \phi^{-1}$ is harmonic on $B_{\phi(x)}(2 R)$ and its total energy over this ball satisfies

$$
E_{B_{\phi(x)}(2 R)}\left(u \circ \phi^{-1}\right)=E_{B_{x}(2 R)}(u) .
$$

Let $\eta(y)=\eta(d(x, y))$ be a smooth cut-off function on $M$ such that $\eta(y)=1$ on $B_{x}(2 R)$ and $\eta(y)=0$ outside the ball $B_{x}(4 R)$. Since $M$ is a Cartan Hadamard manifold with cocompact isometry group, it is easy to see that $|\Delta \eta(y)| \leq c(R)$. Applying Lemma 2.1 and using the fact that $u$ is a $(k, b)$ rough isometry, we have

$$
\begin{aligned}
E_{B_{x}(2 R)}(u) & =\int_{B_{x}(2 R)} e(u)(y) d y \\
& \leq \int_{B_{x}(4 R)} \eta(y) e(u)(y) d y \\
& \leq \frac{1}{2} \int_{B_{x}(4 R)} d^{2}(u(y), u(x)) \Delta \eta(y) d y \\
& \leq c(R)(4 k R+b)^{2} \\
& =c(R, k, b) .
\end{aligned}
$$

By Theorem 2.4.6 in [K-S], we conclude from (2.1) and (2.2) that $u \circ \phi^{-1}$ is uniformly Lipschitz continuous on $B_{\phi(x)}(R)$ with Lipschitz constant bounded by a constant only depending on $R, k$ and $b$. Thus, $u$ is Lipschitz continuous on $B_{x}(R)$ with Lipschitz constant bounded by $c(R, k, b)$. Since $x$ is arbitrary, we conclude that $u$ is uniformly Lipschitz continuous on $M$. The same argument also shows that $v$ is uniformly Lipschitz continuous on $M$.

Since $d(u, v) \leq c$, we may choose $x_{i} \in M$ such that

$$
\lim _{i \rightarrow \infty} d\left(u\left(x_{i}\right), v\left(x_{i}\right)\right)=\sup _{x \in M} d(u(x), v(x)) .
$$


By assumption that both $M$ and $N$ have cocompact isometry groups, there exist isometries $\phi_{i}$ on $M$ and $\psi_{i}$ on $N$ such that $\phi_{i}^{-1}\left(x_{i}\right) \in A$ and $\psi_{i}\left(u\left(x_{i}\right)\right) \in B$, where $A$ and $B$ are two fixed compact subsets of $M$ and $N$ respectively. Consider now

$$
f_{i}=\psi_{i} \circ u \circ \phi_{i}
$$

and

$$
g_{i}=\psi_{i} \circ v \circ \phi_{i}
$$

It is clear that both $f_{i}$ and $g_{i}$ are $(k, b)$ rough isometries for some $(k, b)$ independent of $i$ as both $u$ and $v$ are rough isometries. Let $y_{i}=\phi_{i}^{-1}\left(x_{i}\right) \in A$. The compactness of $A$ implies that, by choosing a subsequence which we also denote by $\left\{y_{i}\right\}, y_{i} \rightarrow y$. Note that

$$
\begin{aligned}
f_{i}\left(y_{i}\right) & =\psi_{i} \circ u \circ \phi_{i}\left(y_{i}\right) \\
& =\psi_{i}\left(u\left(x_{i}\right)\right) \in B
\end{aligned}
$$

and

$$
d\left(f_{i}(y), f_{i}\left(y_{i}\right)\right) \leq k d\left(y, y_{i}\right)+b .
$$

Thus, by the compactness of $B$ and the fact that $y_{i} \rightarrow y$, we conclude that $f_{i}(y)$ is a bounded sequence. Since $N$ is locally compact, we can find a convergent subsequence of $f_{i}(y)$, also denoted by $f_{i}(y)$. Together with the fact that $f_{i}$ are $(k, b)$ rough isometries and $N$ is locally compact, it follows that a subsequence of $f_{i}$, also denoted by $f_{i}$, converges to a map $f$ and the convergence is uniform on compact subsets. Since $f_{i}$ has uniformly bounded Lipschitz constants, $f$ is also a harmonic rough isometry. Using the fact that

$$
d\left(f_{i}, g_{i}\right)=d(u, v) \leq c
$$

by further choosing a subsequence, we conclude that $g_{i}$ also converges to a harmonic rough isometry $g$. Because both $u$ and $v$ have bounded Lipschitz constants, we also see that

$$
\begin{aligned}
d(f(y), g(y))= & \lim _{i \rightarrow \infty} d\left(f_{i}(y), g_{i}(y)\right) \\
= & \lim _{i \rightarrow \infty} d\left(\psi_{i} \circ u \circ \phi_{i}(y), \psi_{i} \circ v \circ \phi_{i}(y)\right) \\
= & \lim _{i \rightarrow \infty} d\left(u \circ \phi_{i}(y), v \circ \phi_{i}(y)\right) \\
\geq & \lim _{i \rightarrow \infty} d\left(u \circ \phi_{i}\left(y_{i}\right), v \circ \phi_{i}\left(y_{i}\right)\right) \\
& \quad-\lim _{i \rightarrow \infty} d\left(u \circ \phi_{i}(y), u \circ \phi_{i}\left(y_{i}\right)\right) \\
& \quad-\lim _{i \rightarrow \infty} d\left(v \circ \phi_{i}(y), v \circ \phi_{i}\left(y_{i}\right)\right) \\
\geq & \lim _{i \rightarrow \infty} d\left(u\left(x_{i}\right), v\left(x_{i}\right)\right) \\
& \quad-2 c \lim _{i \rightarrow \infty} d\left(\phi_{i}(y), \phi_{i}\left(y_{i}\right)\right) \\
= & \sup _{x \in M} d(u(x), v(x)) .
\end{aligned}
$$

However,

$$
d(f, g)=\lim _{i \rightarrow \infty} d\left(f_{i}, g_{i}\right)=d(u, v)
$$


implies that function $d(f, g)$ achieves its maximum at $y$. Since $f$ and $g$ are harmonic and $N$ is an (NPC) space, an argument in [K-S] showed that

$$
\Delta d(f, g) \geq 0 .
$$

Hence the maximum principle implies that $d(f, g)$ must be a constant function. The assumptions that $f$ is a rough isometry and $N$ has negative curvature imply that the image of $f$ does not lie in a geodesic line. The preceding lemma asserts that $u=v$, and theorem is proved.

Corollary 2.4. Let $M^{n}$ and $N^{n}$ be Cartan-Hadamard manifolds with cocompact isometry groups. Suppose that the sectional curvature of $N$ is strictly negative. Let $u$ and $v$ be two harmonic maps from $M$ to $N$ satisfying (A) such that they induce the same boundary map at the infinity. Then $u \equiv v$.

Proof. Since $u$ and $v$ are harmonic maps, they both are continuous maps satisfying (A) between Cartan-Hadamard manifolds of the same dimension. Lemma 10.1' of [M] implies that $u$ and $v$ must map $M$ onto $N$, hence $u$ and $v$ both are rough isometries between $M$ and $N$. Since the sectional curvature of $N$ is negative and $N$ has a cocompact isometry group, the sectional curvature of $N$ satisfies $K_{N} \leq-a^{2}$ for some $a>0$. Therefore, Theorem 2.3 applies and $u=v$.

If $M$ and $N$ are two Cartan-Hadamard manifolds with strongly negative sectional curvatures, i.e., $-b^{2} \leq K_{M}, K_{N} \leq-a^{2}<0$, then a result of Gromov [G] implies that every quasi-conformal diffeomorphism $u: M \rightarrow N$ is uniformly Hölder continuous. Since $u^{-1}$ is also a quasi-conformal diffeomorphism from $N$ onto $M, u^{-1}$ is uniformly Hölder continuous. An easy argument then implies that the map $u$ is a rough isometry between $M$ and $N$ as pointed out in [P 2]. Applying Theorem 2.3 to this setting, we have the following corollary.

Corollary 2.5. Let $M$ and $N$ be Cartan-Hadamard manifolds whose sectional curvatures are strongly negative and both $M$ and $N$ admit cocompact isometry group actions. Then every quasi-conformal harmonic diffeomorphism from $M$ onto $N$ is uniquely determined by its induced boundary map at infinity.

Combining Proposition 0.3 with Corollary 2.4, we obtain the following result which may be view as a generalization of Mostow's rigidity theorem to the trivial groups for the quaternionic hyperbolic spaces or the Cayley hyperbolic plane.

COROLlARY 2.6. Every harmonic rough isometry between a quaternionic hyperbolic space $H_{\mathbb{Q}}^{n}$ and itself or between the Cayley hyperbolic plane $H_{\mathbb{C} a}^{2}$ and itself is an isometry.

Using a recent result of Kleiner and Leeb [K-L], we can also generalize this result to the higher rank case.

THEOREM 2.7. A harmonic rough isometry between an irreducible symmetric space of noncompact type of rank at least two and itself is an isometry.

Proof. Let $M$ be an irreducible symmetric space of noncompact type of rank $r \geq 2$. Let $u$ be a harmonic rough isometry between $M$ and itself. Then by [L-K], there exists an isometry $v$ between $M$ and itself such that the distance between $u$ and $v$ is bounded. Since $M$ is a Cartan Hadamard manifold with cocompact isometry group actions, the argument of Theorem 2.3 implies that the distance between $u$ and $v$ is constant. If $u$ is not identically equal to $v$, then for $p \in M$ let $\gamma$ be the geodesic connecting $u(p)$ and $v(p)$. Let $e_{1}, \ldots, e_{n}$ be an orthonormal frame at $p$ such that $d v\left(e_{1}\right)=\gamma^{\prime}(v(p))$. For each $i=1, \ldots, n$, let us define the vector $X_{i}$ tangent to $M \times M$ by $X_{i}=d u\left(e_{i}\right)+d v\left(e_{i}\right)$. Since $d(u, v)$ is identically constant, the second covariant derivative of $d$ defined on $M \times M$ satisfies $d_{X_{i} X_{i}}=0$. Hence by Proposition 1 of [S-Y], 
the curvature operator of $M$ must satisfy $\left\langle R\left(d v\left(e_{1}\right), d v\left(e_{i}\right)\right) d v\left(e_{i}\right), d v\left(e_{1}\right)\right\rangle=0$ for all $i$. Note that $v$ and hence $d v$ is an isometry. We therefore conclude that the Ricci curvature of $M$ at point $v(p)$ vanishes in the direction $d v\left(e_{1}\right)$. On the other hand, $M$ is an Einstein manifold with negative scalar curvature. In particular, its Ricci curvature is strictly negative everywhere. This contradiction implies that $u$ must be identical to $v$ to begin with. This completes the proof.

3. Existence in Dimension 2. In this section, we turn to address the existence problem for proper harmonic maps between two real hyperbolic planes. In [L-T 3], Tam and the first author established an existence theory for the Dirichlet problem between two real hyperbolic spaces for the class of boundary maps which are $C^{1}$ with nowhere vanishing energy densities. Later, in $[\mathrm{Wg}]$, the second author generalized their existence result to a larger class of boundary maps by allowing some specific type of singularities. In what follows, we restrict ourselves to the situation that both the domain and target are the hyperbolic plane and further generalize these results. As a consequence, we conclude that every real analytic quasi-symmetric map between the unit circle $S^{1}$ and itself admits a (necessarily unique) quasi-conformal harmonic diffeomorphic extension. This partially answers the conjecture of Schoen mentioned in the introduction.

Using the upper half-space model, $\mathbb{R}_{+}^{2}$, for the hyperbolic plane $H_{\mathbb{R}}^{2}$, the rectangular coordinates $(x, y)$ and the polar coordinates $(r, \theta)$ are related by

$$
x=r \cos \theta, \quad y=r \sin \theta .
$$

Also the hyperbolic metric is given by

$$
\begin{aligned}
d s^{2} & =\frac{d x^{2}+d y^{2}}{y^{2}} \\
& =\frac{1}{r^{2} \sin ^{2} \theta} d r^{2}+\frac{1}{\sin ^{2} \theta} d \theta^{2} .
\end{aligned}
$$

For a map $u: H_{\mathbb{R}}^{2} \rightarrow H_{\mathbb{R}}^{2}$, if we use polar coordinates on both domain and target and write $u(r, \theta)=(f(r, \theta), g(r, \theta))$, then the tension field of $u$ is given by

$$
\begin{gathered}
\tau^{1}(u)=r^{2} \Delta_{0} f \sin ^{2} \theta-2 r^{2} \sin ^{2} \theta \cot (g) \frac{\partial g}{\partial r} \frac{\partial f}{\partial r}-\frac{r^{2} \sin ^{2} \theta}{f}\left(\frac{\partial f}{\partial r}\right)^{2} \\
-\frac{\sin ^{2} \theta}{f}\left(\frac{\partial f}{\partial \theta}\right)^{2}-2 \sin ^{2} \theta \cot (g) \frac{\partial g}{\partial \theta} \frac{\partial f}{\partial \theta}
\end{gathered}
$$

and

$$
\begin{gathered}
\tau^{2}(u)=r^{2} \Delta_{0} g \sin ^{2} \theta+\frac{r^{2} \sin ^{2} \theta \cot (g)}{f^{2}}\left(\frac{\partial f}{\partial r}\right)^{2}-r^{2} \sin ^{2} \theta \cot (g)\left(\frac{\partial g}{\partial r}\right)^{2} \\
+\frac{\sin ^{2} \theta \cot (g)}{f^{2}}\left(\frac{\partial f}{\partial \theta}\right)^{2}-\sin ^{2} \theta \cot (g)\left(\frac{\partial g}{\partial \theta}\right)^{2}
\end{gathered}
$$

Here the Laplacian $\Delta_{0}$ is the Laplacian with respect to the Euclidean metric on $\mathbb{R}^{2}$.

THEOREM 3.1. Let $f$ be a map from the extended real line $\overline{\mathbb{R}}$ onto itself. Suppose that $f$ is $C^{1}$ and has nowhere vanishing energy density on $\overrightarrow{\mathbb{R}}$ except for a finite set of points $\left\{p_{1}, \ldots, p_{k}\right\}$. Assume that near each point $p_{i}$, after choosing $p_{i}$ and $f\left(p_{i}\right)$ as origins for the domain and target, the map is of the form $f(r, 0)=\left(r^{\alpha} h_{1}(r), 0\right)$ 
and $f(r, \pi)=\left(r^{\alpha} h_{2}(r), \pi\right)$, for some $\alpha>0$, and for positive $C^{1}$ functions $h_{1}$ and $h_{2}$ satisfying $h_{1}(0)=h_{2}(0)$ and $h_{1}^{\prime}(0)=h_{2}^{\prime}(0)$. Then there is a proper harmonic map $u: H_{\mathbb{R}}^{2} \rightarrow H_{\mathbb{R}}^{2}$ such that $u=f$ at the infinity. Moreover, if $f$ is also a homeomorphism, then $u$ is a quasi-conformal diffeomorphism.

Proof. We only give the proof for the case $k=1$ as the proof for the general case is similar. We claim that for given $\epsilon>0$ there exists an extension of $f$ to a proper map $F$ from $H_{\mathbb{R}}^{2}$ into $H_{\mathbb{R}}^{2}$ satisfying the following properties.

(1) The Jacobian of the map $F$ satisfies $J(F) \geq C>0$ for some constant $C$ independent of $\epsilon$ outside a compact subset of $H_{\mathbb{R}}^{2}$.

(2) The energy density function of $F$ satisfies $e(F) \leq c$.

(3) The tension vector field of $F$ satisfies $\sup _{y \leq \delta(\epsilon)}\|\tau(F)\|(x, y) \leq \epsilon$, where $\delta(\epsilon)>$ 0 .

Once this claim is established, the existence of the harmonic map $u$ then follows by applying the argument of Theorem 6.4 in [L-T 3]. Moreover, from the argument one concludes that the map $u$ has bounded energy density and the Jacobian of $u$ is nowhere vanishing outside a compact subset. If the boundary map $f$ is a homeomorphism, then one sees that the Jacobian of $u$ is nowhere vanishing everywhere by Theorem 7.1 in $[\mathrm{J}]$. Hence $u$ becomes a diffeomorphism. Since $u$ has bounded energy density, from $[\mathrm{W}]$ one knows that $u$ is quasi-conformal.

To verify the claim, without loss of generality, we may assume that the point $p_{1}$ and $f\left(p_{1}\right)$ are the origins of the domain and target. Also, we may assume that in terms of polar coordinates, when $0 \leq r \leq 1, f(r, 0)=\left(r^{\alpha} h_{1}(r), 0\right)$ and $f(r, \pi)=\left(r^{\alpha} h_{2}(r), \pi\right)$, where $h_{1}$ and $h_{2}$ are $C^{1}$ positive functions and $h_{1}(0)=h_{2}(0), h_{1}^{\prime}(0)=h_{2}^{\prime}(0)$. Let $v(r, \theta)$ be the function defined on the upper half plane such that $\Delta_{0} v=0, v(r, 0)=h_{1}(r)$ and $v(r, \pi)=h_{2}(r)$. Let the function $g$ solve the following ordinary differential equation

$$
g^{\prime \prime} \sin ^{2} \theta-\left(g^{\prime}\right)^{2} \sin ^{2} \theta \cot (g)+\alpha^{2} \sin ^{2} \theta \cot (g)=0
$$

with boundary conditions $g(0)=0$ and $g(\pi)=\pi$. By [Wg], such $g$ exists and $g$ is smooth on $[0, \pi]$ with $0<c_{1} \leq g^{\prime} \leq c_{2}$ on $[0, \pi]$. We now extend the boundary map $f$ to a map $u$ defined on the half disk $0<r \leq 1,0<\theta<\pi$ by

$$
u(r, \theta)=\left(r^{\alpha} v(r, \theta), g(\theta)\right) .
$$

Since $h_{1}$ and $h_{2}$ are positive, we know $v$ is also positive. So $u$ defines a map from the half disk into $H^{2}$. Using the fact that $0<c_{1} \leq g^{\prime} \leq c_{2}$ and $v$ is $C^{1}$ up to the boundary and positive, one verifies that the energy density $e(u)$ of $u$ is bounded and the Jacobian $J(u)$ satisfies $J(u) \geq c_{3}>0$ for $0<r \leq c_{4} \leq 1$. In the following, we estimate the tension field of $u$. By (3.1) and (3.4), noting that $g$ only depends on $\theta$, we have

$$
\begin{aligned}
\tau^{1}(u)= & r^{2} \Delta_{0}\left(r^{\alpha} v\right) \sin ^{2} \theta-\frac{r^{2} \sin ^{2} \theta}{r^{\alpha} v}\left(\frac{\partial\left(r^{\alpha} v\right)}{\partial r}\right)^{2} \\
& \quad-\frac{\sin ^{2} \theta}{r^{\alpha} v}\left(\frac{\partial\left(r^{\alpha} v\right)}{\partial \theta}\right)^{2}-2 \sin ^{2} \theta \cot (g) \frac{\partial g}{\partial \theta} \frac{\partial\left(r^{\alpha} v\right)}{\partial \theta} \\
= & -r^{\alpha}\left(\frac{r^{2}}{v}\left(\frac{\partial v}{\partial r}\right)^{2}+\frac{1}{v}\left(\frac{\partial v}{\partial \theta}\right)^{2}+2 \cot (g) g^{\prime} \frac{\partial v}{\partial \theta}\right) \sin ^{2} \theta .
\end{aligned}
$$

Since $v$ is a harmonic function and its boundary value is $C^{1}$ and positive, we have 
$v \geq c>0,\left|\nabla_{0} v\right| \leq c($ see $[\mathrm{G}-\mathrm{T}])$. Therefore,

$$
\left|\frac{\partial v}{\partial r}\right| \leq c, \quad\left|\frac{\partial v}{\partial \theta}\right| \leq c r
$$

Using (3.6) and the fact that $0<c_{1} \leq g^{\prime} \leq c_{2}$, we conclude from (3.5) that

$$
\left|\tau^{1}(u)\right| \leq c r^{\alpha+1} \sin \theta .
$$

From (3.2) and (3.4), we have by using (3.3)

$$
\begin{aligned}
\tau^{2}(u)= & g^{\prime \prime} \sin ^{2} \theta+\frac{r^{2} \sin ^{2} \theta \cot (g)}{r^{2 \alpha} v^{2}}\left(\frac{\partial\left(r^{\alpha} v\right)}{\partial r}\right)^{2} \\
& +\frac{\sin ^{2} \theta \cot (g)}{r^{2 \alpha} v^{2}}\left(\frac{\partial\left(r^{\alpha} v\right)}{\partial \theta}\right)^{2}-\left(g^{\prime}\right)^{2} \sin ^{2} \theta \cot (g) \\
= & \left(2 \alpha r v \frac{\partial v}{\partial r}+r^{2}\left|\nabla_{0} v\right|^{2}\right) \frac{\sin ^{2} \theta \cot (g)}{v^{2}}
\end{aligned}
$$

However, $v$ is $C^{1}$ up to the boundary and its boundary value is positive, we conclude from (3.8) that

$$
\left|\tau^{2}(u)\right| \leq c r \sin \theta
$$

Therefore, by (3.7) and (3.9), we have

$$
\begin{aligned}
\|\tau(u)\|^{2} & =\frac{1}{\left(r^{\alpha} v\right)^{2} \sin ^{2}(g)}\left|\tau^{1}(u)\right|^{2}+\frac{1}{\sin ^{2}(g)}\left|\tau^{2}(u)\right|^{2} \\
& \leq c r^{2} \frac{\sin ^{2} \theta}{\sin ^{2}(g)}+c r^{2} \frac{\sin ^{2} \theta}{\sin ^{2}(g)} \\
& \leq c r^{2}
\end{aligned}
$$

In conclusion, we can choose $c_{5}(\epsilon)>0$ sufficiently small such that for $0<r \leq c_{5} \leq 1$ the map $u$ satisfies $\|\tau(u)\| \leq \epsilon / 2$. Note that for $r \geq c_{5} / 2$ the boundary map $f$ is $C^{1}$ with nowhere vanishing energy density. In particular, by [L-T 3], there exists a proper harmonic extension $w$ from $H^{2}$ into $H^{2}$ such that $w$ is $C^{1}$ up to the boundary and $w=f$ on the boundary for $r \geq c_{5} / 2$. So the energy density $e(w)$ of $w$ satisfies $e(w) \leq c_{6}$ which may depend on $\epsilon$. On the other hand, from [L-T 1] we know that the map $w$ is conformal and $e(w)=2$ near the boundary. Thus, we may choose $\delta_{1}$ small such that $J(w) \geq 1$ and $e(w) \leq 3$ for $y \leq \delta_{1}$. Finally, let $\phi$ be a smooth function with compact support in the half disk $0 \leq r \leq c_{5}, 0 \leq \theta \leq \pi$ such that $0 \leq \phi(r, \theta) \leq 1$ and $\phi(r, \theta)=1$ for $r \leq c_{5} / 2$. We now define map $\bar{F}$ and in terms of rectangular coordinate system $F(x, y)=\phi(x, y) u(x, y)+(1-\phi(x, y)) w(x, y)$. Clearly, $F$ is welldefined provided that $y$ is small and $F=u$ for $r \leq c_{5} / 2$ and $F=w$ for $r \geq c_{5}$. Using the fact that both $u$ and $w$ are $C^{1}$ up to boundary, we conclude from the computation in [L-T 3] that

$$
\|\tau(F)\| \leq \phi\|\tau(u)\|+(1-\phi)\|\tau(w)\|+O(y)
$$

when $c_{5} / 2 \leq r \leq c_{5}$ and $y$ is small. Therefore, by choosing $\delta$ even smaller, we have $\|\tau(F)\| \leq \epsilon$ when $y \leq \delta$. From $J(u) \geq c_{3}$ and $J(w) \geq 1$, and $e(u) \leq c$ and $e(w) \leq 3$ when $y$ is small, one easily sees that $J(F) \geq c>0$ and $e(F) \leq c$, where $c$ is independent of $\epsilon$. In conclusion, we have verified such $F$ satisfies the claim. The theorem is thus proved. 
For a real analytic quasi-symmetric map between $S^{1}$ and itself, Theorem 3.1 is clearly applicable.

COROLlARY 3.2. Every real analytic quasi-symmetric map between $S^{1}$ and itself can be extended to a quasi-conformal harmonic diffeomorphism between $H_{\mathbb{R}}^{2}$.

4. Appendix. In this section, we will give a proof of proposition 1.3. We start with the following lemma.

LEMMA 4.1. Let $l$ be a geodesic line containing a point $R$ in $\mathbb{H}^{2}$. Let $Q$ be an interior point of the triangle $\triangle P R S$ in $\mathbb{H}^{2}$, where $P$ is a point on $l$. Then $d(Q, S)$ is an increasing function of $d(P, R)$ as $P$ moves along $l$ with $d(P, Q), d(Q, R), d(R, S)$ and $d(S, P)$ fixed.

Proof. Let $p=d(P, Q), q=d(Q, R), r=d(R, S), s=d(S, P)$, and $d(P, R)=t$. Denote the angles $\angle P R Q=\theta, \angle P R S=\alpha, \angle R P Q=\phi$ and $\angle R P S=\beta$. We will show that $\alpha-\theta$ is an increasing function of $t$. Note that

$$
\cosh t \cosh q-\cosh p=\cos \theta \sinh t \sinh q,
$$

hence

$$
\begin{aligned}
\theta_{t} & =-\frac{\cosh t \cosh p-\cosh q}{\sinh ^{2} t \sinh q \sin \theta} \\
& =-\frac{\sinh p \cos \phi}{\sinh t \sinh q \sin \theta} .
\end{aligned}
$$

Let $y=d(Q, l)$ and $x=d\left(P, \pi_{l}(Q)\right)$. Then direct computation gives

$$
\sinh q \sin \theta=\sinh y,
$$

and

$$
\sinh p|\cos \phi|=\sinh x \cosh y .
$$

Similarly, if we let $\bar{y}=d(S, l)$ and $\bar{x}=d\left(P, \pi_{l}(S)\right)$, then

$$
\begin{gathered}
\alpha_{t}=-\frac{\sinh s \cos \beta}{\sinh t \sinh r \sin \alpha}, \\
\sinh r \sin \alpha=\sinh \bar{y}
\end{gathered}
$$

and

$$
\sinh s|\cos \beta|=\sinh \bar{x} \cosh \bar{y} .
$$

Thus, if $\beta \geq \pi / 2$ and $\phi \leq \pi / 2$, then

$$
\alpha_{t}-\theta_{t} \geq 0
$$

If both $\beta$ and $\phi$ are less than $\pi / 2$, then

$$
\alpha_{t}-\theta_{t}=\frac{1}{\sinh t}\left(\frac{\sinh x \cosh y}{\sinh y}-\frac{\sinh \bar{x} \cosh \bar{y}}{\sinh \bar{y}}\right) .
$$

In this case, let $\delta$ be the geodesic segment joining $S$ and $\pi_{l}(S)$. It is clear that $\delta$ intersects $P Q$ at some point $T$. Define $z=d(T, l)$, then $z \leq \bar{y}$. Direct computation yields

$$
\frac{\sinh x \cosh y}{\sinh y}=\frac{\sinh \bar{x} \cosh z}{\sinh z} .
$$


Combining (4.1) and (4.2) yields,

$$
\alpha_{t}-\theta_{t}=\frac{\sinh \bar{x}}{\sinh t}\left(\frac{1}{\tanh z}-\frac{1}{\tanh \bar{y}}\right) \geq 0 .
$$

Finally, we consider the case when both $\phi$ and $\beta$ are greater than $\pi / 2$, then

$$
\alpha_{t}-\theta_{t}=\frac{1}{\sinh t}\left(-\frac{\sinh x \cosh y}{\sinh y}+\frac{\sinh \bar{x} \cosh \bar{y}}{\sinh \bar{y}}\right) .
$$

Let $\gamma$ be the geodesic line passing through $S$ and $\pi_{l}(S)$. It is clear that $\gamma$ does not intersect $P Q$, but the line passing through $P$ and $Q$ intersects $\gamma$ at some point $H$. If we define $h=d(H, l)$, then $h \geq \bar{y}$. Direct computation yields

$$
\frac{\sinh x \cosh y}{\sinh y}=\frac{\sinh \bar{x} \cosh h}{\sinh h} .
$$

This combining with (4.3) implies,

$$
\alpha_{t}-\theta_{t}=\frac{\sinh \bar{x}}{\sinh t}\left(-\frac{1}{\tanh h}+\frac{1}{\tanh \bar{y}}\right) \geq 0 .
$$

In conclusion, $\alpha_{t}-\theta_{t} \geq 0$ and $\alpha-\theta$ is increasing. Now it is easy to see that $d(Q, S)$ is increasing with respect to $t$, and the lemma is proved.

Let $\mathbb{H}_{a}^{2}$ denote the two dimensional model space of constant curvature $-a^{2}$. So it is the standard $\mathbb{R}^{2}$ when $a=0$ and $\mathbb{H}^{2}$ if $a=1$. The following quadrilateral comparison property for a metric space $X$ with curvature upper bounds follows from a substantially more general theorem due to Reshetnyak $[R]$. A proof of the case that $X$ is an (NPC) space has been included in [K-S]. The same proof also works for the more general case that $K_{X} \leq-a^{2}$ by noticing Lemma 4.1 . We shall not reproduce the details here.

Proposition 4.2. Let $(X, d)$ be a metric space with $K_{X} \leq-a^{2}$ for some $a \geq 0$. Let $P, Q, R$ and $S$ be an ordered sequence of four points in $X$. Then there exist points $\bar{P}, \bar{Q}, \bar{R}$ and $\bar{S}$ in $\mathbb{H}_{a}^{2}$ so that they form the consecutive vertices of a convex quadrilateral in $\mathbb{H}_{a}^{2}$ with $d(P, Q)=d(\bar{P}, \bar{Q}), d(Q, R)=d(\bar{Q}, \bar{R}), d(R, S)=d(\bar{R}, \bar{S})$, $d(S, P)=d(\bar{S}, \bar{P}), d(P, R) \leq d(\bar{P}, \bar{R})$ and $d(Q, S) \leq d(\bar{Q}, \bar{S})$.

Such a convex quadrilateral formed by $\bar{P}, \bar{Q}, \bar{R}$ and $\bar{S}$ in $\mathbb{H}_{a}^{2}$ is called the comparison quadrilateral corresponding to the ordered sequence of points $P, Q, R$ and $S$ in $X$.

Proposition 4.3. Let $(X, d)$ be a metric space with $K_{X} \leq-a^{2}$ for some $a \geq 0$. Let $P, Q, R$ and $S$ be an ordered sequence of four points in $X$. Let $\bar{P}, \bar{Q}, \bar{R}$ and $\bar{S}$ be the consecutive vertices of a comparison quadrilateral in $\mathbb{H}_{a}^{2}$. For any given $0 \leq \lambda, \mu \leq 1$, let us define $P_{\lambda}\left(\bar{P}_{\lambda}\right)$ to be the point which is a fraction $\lambda$ of the way from $P(\overline{\bar{P}})$ to $S(\bar{S})$ on the geodesic $\gamma_{P, S}\left(\gamma_{\bar{P}, \bar{S}}\right)$. Similarly, let $Q_{\mu}\left(\bar{Q}_{\mu}\right)$ be the point which is a fraction $\mu$ of the way from $Q(\bar{Q})$ to $R(\bar{R})$ on the geodesic $\gamma_{Q, R}\left(\gamma_{\bar{Q}, \bar{R}}\right)$. Then

$$
d\left(P_{\lambda}, Q_{\mu}\right) \leq d\left(\overline{P_{\lambda}}, \overline{Q_{\mu}}\right)
$$

Proof. A proof of the case $a=0$ has been given in [K-S]. We only need to consider the case $a>0$. Without loss of generality, we may assume that $a=1$. Let us first show that

$$
d\left(P, Q_{\mu}\right) \leq d\left(\bar{P}, \bar{Q}_{\mu}\right)
$$


Let $p=d(P, Q), q=d(Q, R), r=d(R, S)$ and $s=d(S, P)$. Also, let $\triangle A B C$ be a triangle in $\mathbb{H}^{2}$ such that $d(A, B)=p, d(B, C)=q$ and $d(C, A)=d(P, R)$. Let $B_{\mu}$ be the point on the side determined by $B$ and $C$ such that $d\left(B_{\mu}, B\right)=\mu q$. Then we have

$$
d\left(P, Q_{\mu}\right) \leq d\left(A, B_{\mu}\right)
$$

However,

$$
\begin{aligned}
\cosh d\left(A, B_{\mu}\right) & =\cosh p \cosh (\mu q)-\frac{\sinh (\mu q)}{\sinh q}(\cosh p \cosh q-\cosh d(A, C)) \\
& \leq \cosh p \cosh (\mu q)-\frac{\sinh (\mu q)}{\sinh q}(\cosh p \cosh q-\cosh d(\bar{P}, \bar{R})) \\
& =\cosh d\left(\bar{P}, \bar{Q}_{\mu}\right)
\end{aligned}
$$

therefore

$$
d\left(P, Q_{\mu}\right) \leq d\left(A, B_{\mu}\right) \leq d\left(\bar{P}, \overline{Q_{\mu}}\right)
$$

Analogously, we conclude that

$$
d\left(S, Q_{\mu}\right) \leq d\left(\bar{S}, \overline{Q_{\mu}}\right) .
$$

Let $\triangle D E F$ be a triangle in $\mathbb{H}^{2}$ such that $d\left(P, Q_{\mu}\right)=d(D, E), d\left(Q_{\mu}, S\right)=d(E, F)$ and $d(F, E)=s$. Let $D_{\lambda}$ be the point on the side determined by $D$ and $F$ such that $d\left(D_{\lambda}, D\right)=\lambda s$. Then we have

$$
d\left(P_{\lambda}, Q_{\mu}\right) \leq d\left(E, D_{\lambda}\right)
$$

However, since

$$
\begin{aligned}
& \cosh d\left(E, D_{\lambda}\right) \\
& =\cosh d(D, E) \cosh (\lambda s)-\frac{\sinh (\lambda s)}{\sinh s}(\cosh d(D, E) \cosh s-\cosh d(E, F)) \\
& =\cosh d\left(P, Q_{\mu}\right)\left(\cosh (\lambda s)-\frac{\sinh (\lambda s)}{\sinh s} \cosh s\right)+\frac{\sinh (\lambda s)}{\sinh s} \cosh d\left(S, Q_{\mu}\right) \\
& \leq \cosh d\left(\bar{P}, \overline{Q_{\mu}}\right)\left(\cosh (\lambda s)-\frac{\sinh (\lambda s)}{\sinh s} \cosh s\right)+\frac{\sinh (\lambda s)}{\sinh s} \cosh d\left(\bar{S}, \overline{Q_{\mu}}\right) \\
& =\cosh d\left(\overline{P_{\lambda}}, \overline{Q_{\mu}}\right)
\end{aligned}
$$

hence

$$
d\left(P_{\lambda}, Q_{\mu}\right) \leq d\left(E, D_{\lambda}\right) \leq d\left(\bar{P}_{\lambda}, \overline{Q_{\mu}}\right)
$$

and the proof is complete.

We are now ready to prove Proposition 1.3.

Proof of Proposition 1.3. Without loss of generality, we may assume that $L$ is a broken geodesic connecting points $p_{1}, p_{2}, \ldots, p_{k}$ in order. The general case then follows from a simple approximation argument. In what follows, we will show that for each geodesic segment $\beta_{i}$ connecting $p_{i}$ and $p_{i+1}, i=1, \ldots, k-1$, one has

$$
\left|\pi_{l}\left(\beta_{i}\right)\right| \leq \frac{\left|\beta_{i}\right|}{\cosh (a R)},
$$


and this will imply that

$$
\begin{aligned}
\left|\pi_{l}(L)\right| & \leq \sum_{i=1}^{k-1}\left|\pi_{l}\left(\beta_{i}\right)\right| \\
& \leq \sum_{i=1}^{k-1} \frac{\left|\beta_{i}\right|}{\cosh (a R)} \\
& =\frac{|L|}{\cosh (a R)} .
\end{aligned}
$$

For simplicity of notations, we call $\beta_{i}$ to be $\beta$ and its end points $g$ and $q$. Let $j=\pi_{l}(q)$ and $t=\pi_{l}(g)$. Consider the quadrilateral formed by the ordered sequence of points $g, q, j, t$ and the corresponding comparison quadrilateral with consecutive vertices $G$, $Q, J, T$ in $\mathbb{H}_{a}^{2}$. Let $\gamma$ be the geodesic line passing through $J$ and $T$. We first note that the angles satisfy

$$
\angle G T J \geq \pi / 2, \quad \text { and } \quad \angle Q J T \geq \pi / 2 .
$$

In fact, for any point $H$ on the segment $T J$, write $d(H, T)=\mu d(T, J)$ for some $0 \leq$ $\mu \leq 1$. Pick up a point $h$ on the side determined by $t$ and $j$ such that $d(h, t)=\mu d(t, j)$. Then Proposition 4.3 implies that

$$
\begin{aligned}
d(G, H) & \geq d(g, h) \\
& \geq d(g, t) \\
& =d(G, T) .
\end{aligned}
$$

Since $H$ is arbitrary on $T J$, one concludes that $\angle G T J \geq \pi / 2$. Similarly, one also conludes that $\angle Q J T \geq \pi / 2$. Therefore, we may find points $M$ and $N$ on the side $G Q$ such that $T=\pi_{\gamma}(M)$ and $J=\pi_{\gamma}(N)$. For a point $Y$ on the segment $M N$, it is then clear that $\pi_{\gamma}(Y)=F \in T J$. Let $y$ and $f$ be the points on the segments $g q$ and $t j$ respectively such that $d(y, g)=d(Y, G)$ and $d(f, t)=d(F, T)$. Then

$$
\begin{aligned}
R & \leq d(y, f) \\
& \leq d(Y, F) \\
& =d(Y, \gamma) .
\end{aligned}
$$

Therefore, the segment $M N$ is at least of $R$ distance from $\gamma$. We then conclude that

$$
\left|\pi_{\gamma}(M N)\right| \leq \frac{|M N|}{\cosh R}
$$

However, $\pi_{\gamma}(M N)=T J$, hence

$$
\begin{aligned}
\left|\pi_{l}(g q)\right| & =|t j| \\
& =|T J| \\
& =\left|\pi_{\gamma}(M N)\right| \\
& \leq \frac{|M N|}{\cosh R} \\
& \leq \frac{|G Q|}{\cosh R} \\
& =\frac{|g q|}{\cosh R},
\end{aligned}
$$


which completes the proof.

\section{REFERENCES}

[C] S. Y. Cheng, Liouville theorem for harmonic maps, Proc. Symposia Pure Math., 36 (1980), pp. 147-151.

[D 1] H. Donnelly, Dirichlet problem at infinity for harmonic maps-rank one symmetric spaces, TAMS, 344 (1994), pp. 713-736.

[D 2] H. Donnelly, Harmonic maps between rank one symmetric spaces - regularity at ideal boundary, preprint.

[E] P. EBerlein, Geodesic rigidity in compact non-positively curved manifolds, Trans. AMS, 268 (1981), pp. 411-443.

[G] M. Gromov, Pseudo-holomorphic curves in symplectic manifolds, Inven. Math., 82 (1985), pp. 307-347.

[G-T] D. Gilbarg And N. S. Trudinger, Elliptic partial differential equations of second order, Springer-Verlag, Berlin-Heidelberg-New York, 1977.

$[\mathbf{J}]$ J. Jost, Harmonic mappings between surfaces, Lecture Notes in Math. 1062, Springer, 1984.

[K-L] B. KLEINER AND B. Leeb, Rigidity of quasi-isometries for symmetric spaces of higher rank, preprint.

[K-S] N. KorevaAR And R. Schoen, Sobolev spaces and harmonic maps from metric space targets, Comm. Anal. Geom., 1 (1993), pp. 561-659.

[L-T 1] P. Li AND L. F. TAM, The heat equation and harmonic maps of complete manifolds, Invent. Math., 105 (1991), pp. 1-46.

[L-T 2] P. Li AND L. F. TAM, Uniqueness and regularity of proper harmonic maps, Ann. Math., 136 (1992), pp. 169-203.

[L-T 3] P. Li AND L. F. TAM, Uniqueness and regularity of proper harmonic maps II, Indiana J. Math., 42 (1993), pp. 591-635.

[M] G. D. Mostow, Strong rigidity of locally symmetric spaces, Annals of Math. Studies no. 78, Princeton University Press, 1973.

[P 1] P. PANsu, Métriques de Carnot-Carathéodory et quasiisométries des espaces symétriques de range un, Ann. Math., 129 (1989), pp. 1-60.

[P 2] P. PAnsu, Quasi-conformal mappings and manifolds of negative curvature, in Lecture Notes of Math., 1201, pp. 212-229.

[R] Y. RESHETNYAK, Inextensible mappings in a space of curvature no greater than K, Siberian. Math. Jour., 9 (1968), pp. 918-927.

[S] R. SCHOEN, The role of harmonic mappings in rigidity and deformation problems, "Complex Geometry" Lecture notes in pure and applied mathematics, edited by G. Komatsu and Yusuke Sakane, 143 (1993), Marcel Dekker, Inc., New York · Basel · Hong Kong, pp. 179-200.

[S-Y] R. Schoen AND S. T. YAU, Compact group actions and the topology of manifolds with nonpositive curvature, Topology, 18 (1979), pp. 361-380.

[T-W] L. F. TAM AND T. WAN, Quasi-conformal harmonic diffeomorphisms and the universal Teichmüller space, J. Diff. Geom., 42 (1995), pp. 368-410.

[T] P. TUKIA, Quasi-conformal extensions of quasi-symmetric mappings compatible with a Möbius group, Acta Math., 154 (1985), pp. 153-193.

[W] T. WAN, Constant mean curvature surface, harmonic maps and universal Teichmüller space, J. Diff. Geom., 35 (1992), pp. 643-657.

[Wa] A. WALD, Begründung einer koordinatenlosen Differentialgeometrie der Flächen, Ergebnisse eines mathematischen Kooloquiums, 7 (1935), pp. 2-46.

[Wg] J. WANG, The heat flow and harmonic maps between complete manifolds, J. Geom. Anal., to appear. 